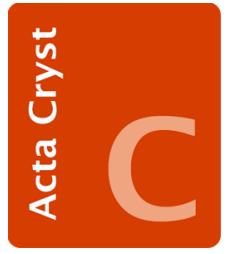

STRUCTURAL

CHEMISTRY

Volume 76 (2020)

Supporting information for article:

Synthesis and X-ray crystal structures of zinc(II) and mercury(II) complexes based on $N, N^{\prime}$-(cyclohexane-1,2-diylidene)bis(4-fluorobenzoylhydrazide)

Al-Ameen Bariz OmarAli, Ahmed Jasim M. Al-Karawi, Adil A. Awad, Necmi Dege, Sevgi Kansız, Erbil Agar, Zaman Ahmed Hussein and Iman Rajab Mohammed 

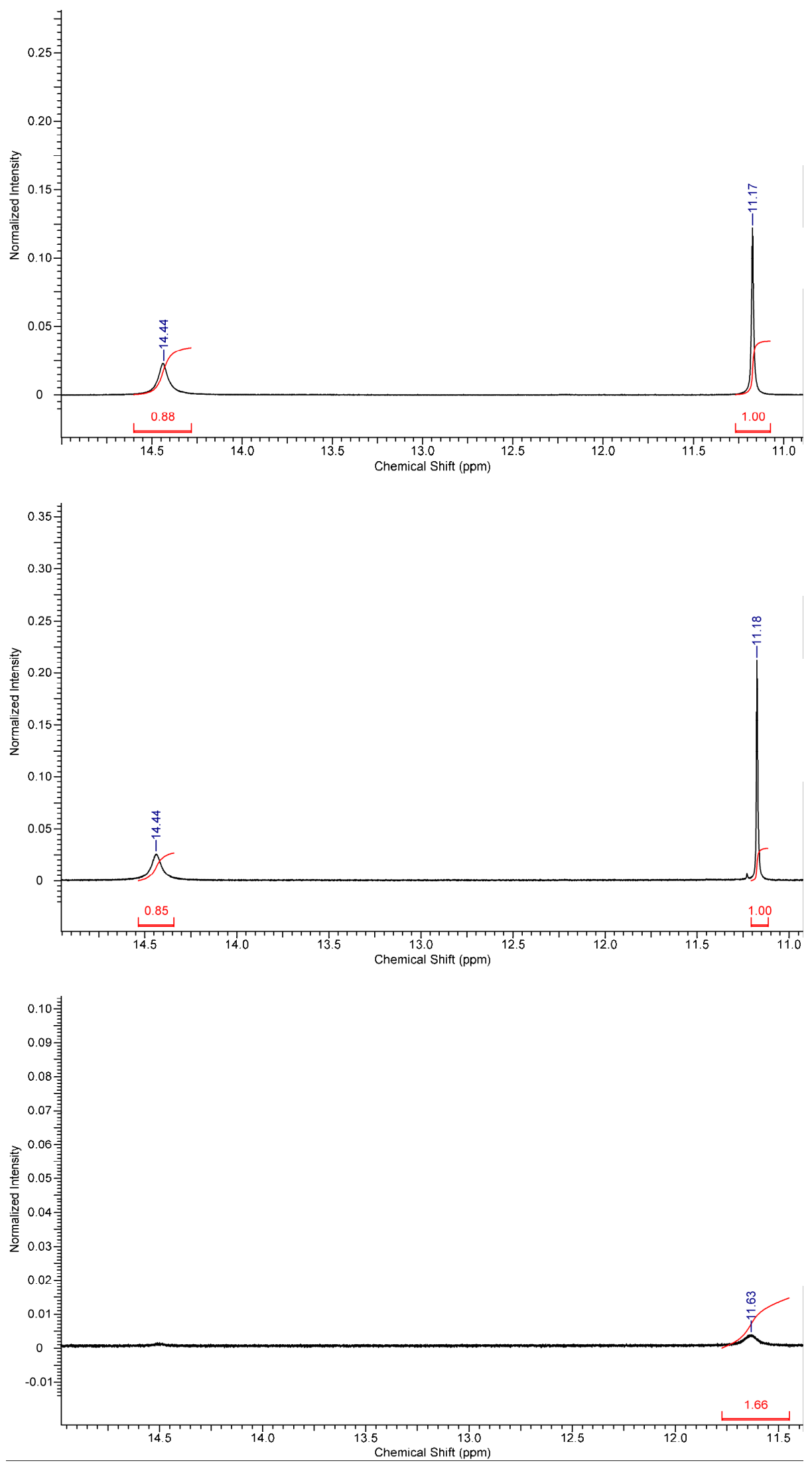

Figure S1

${ }^{1}$ HNMR spectra of $\mathbf{L}^{\mathbf{F}}$ (Top), $\mathbf{1}$ (Middle) and $\mathbf{2}$ (Bottom), showing the keto-enol tautomerism 


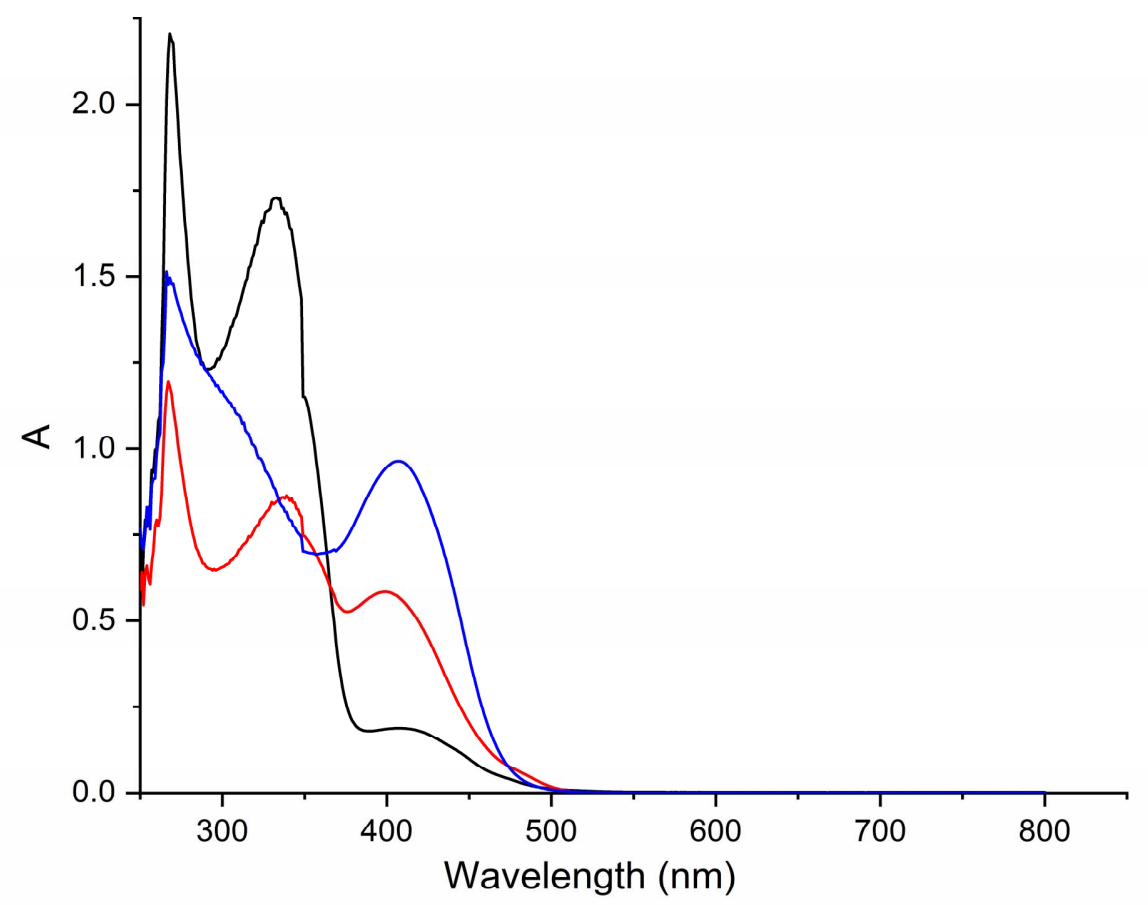

\section{Figure S2}

UV-Vis spectra of the prepared compounds: $\mathbf{L}^{\mathbf{F}}$ (Black line), $\mathbf{1}$ (Red line) and $\mathbf{2}$ (Blue line)

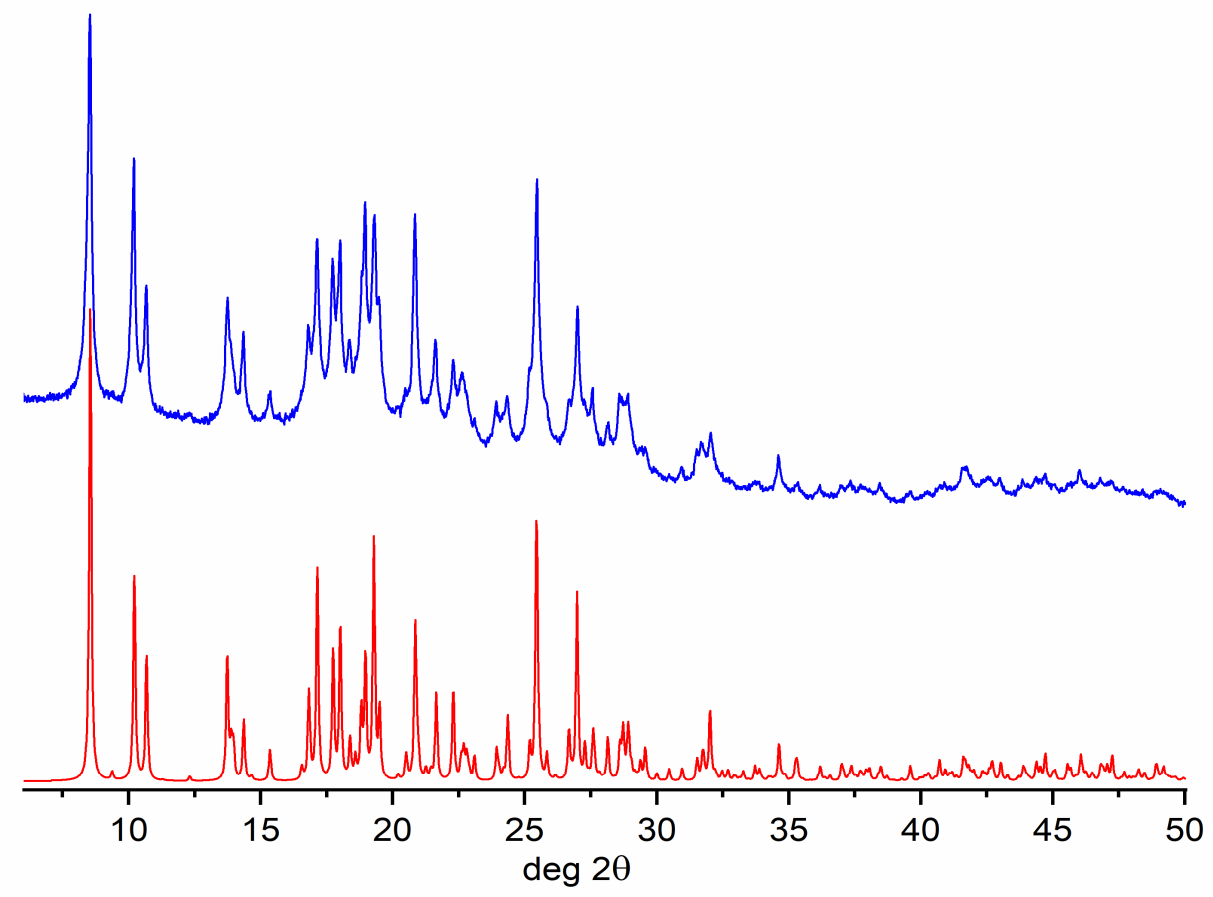

\section{Figure S3}

X-ray diffraction pattern of 1: experimental diffraction pattern (Blue line); calculated pattern from single crystal data (Red line) 


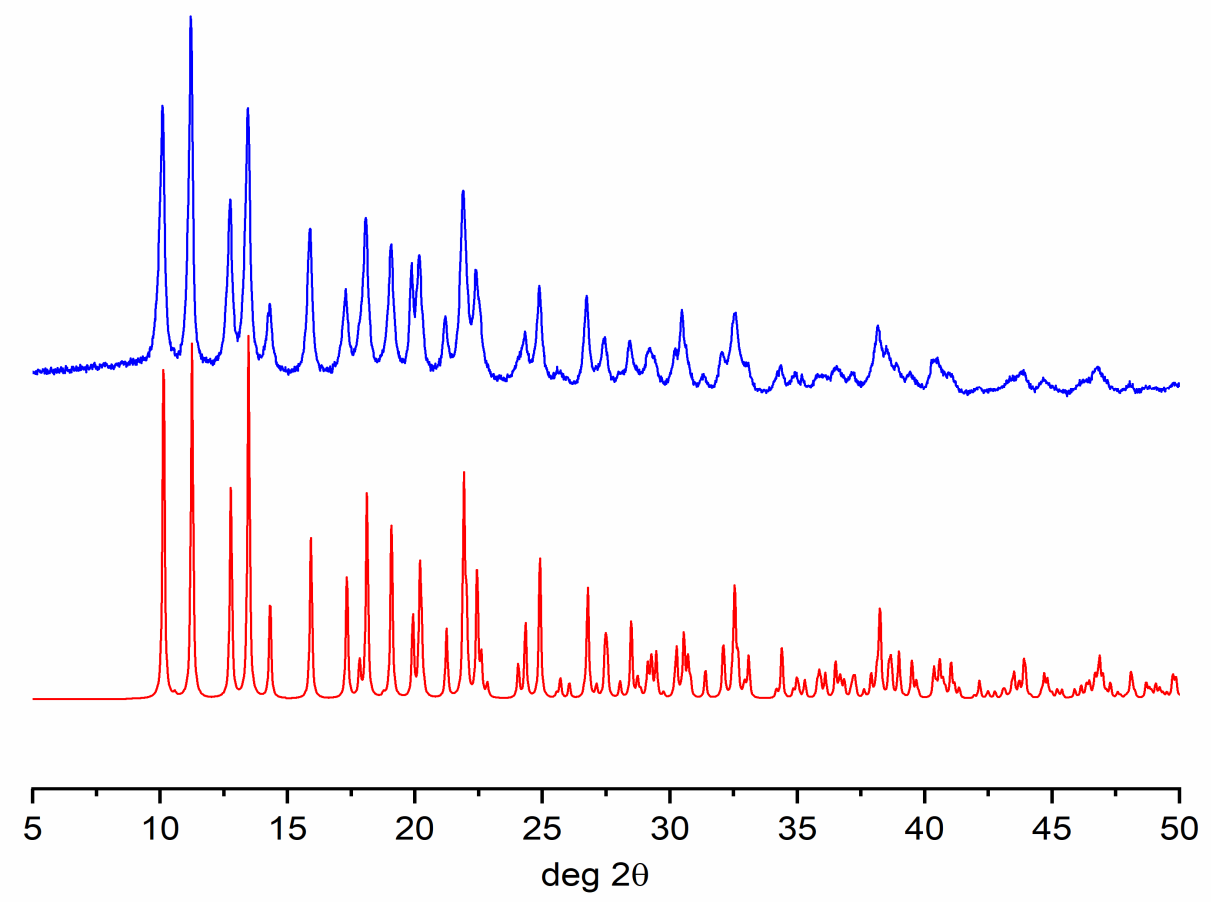

\section{Figure S4}

X-ray diffraction pattern of 2: experimental diffraction pattern (Blue line); calculated pattern from single crystal data (Red line) 